\title{
Concurrent chemoradiotherapy for stage III non-small cell lung cancer: correct clinical management as the basis to move beyond
}

\author{
Guilherme Jorge Costa ${ }^{1}$, Carlos Gil Ferreira ${ }^{2}$, Luiz Claudio Santos Thuler $^{3}$ \\ ${ }^{1}$ Instituto de Medicina Integral Prof. Fernando Figueira (IMIP) and Hospital de Câncer de Pernambuco, Recife, Pernambuco, Brazil; ${ }^{2}$ Oncoclinicas \\ Institute for Research and Education, São Paulo, Brazil; ${ }^{3}$ Instituto National de Cancer (INCA), Rio de Janeiro, Brazil \\ Correspondence to: Luiz Claudio Santos Thuler. Instituto National de Cancer (INCA), Rua André Cavalcanti, 37/Anexo-3o andar Centro CEP 20231- \\ 050, Rio de Janeiro, RJ, Brazil. Email: lthuler@gmail.com. \\ Comment on: Deek MP, Kim S, Ahmed I, et al. Prognostic Impact of Missed Chemotherapy Doses During Chemoradiation Therapy for Non-Small \\ Cell Lung Cancer. Am J Clin Oncol 2018;41:362-6.
}

Submitted Sep 29, 2018. Accepted for publication Oct 11, 2018.

doi: $10.21037 / \mathrm{atm} .2018 .10 .38$

View this article at: http://dx.doi.org/10.21037/atm.2018.10.38

Lung cancer (LC) is the most common type of cancer and the principal cause of death from cancer worldwide. In 2018, data from Globocan estimated over 2.1 millions new cases of LC were reported, with 1.8 million deaths globally (1). Smoking is main cause of LC, and almost $85-90 \%$ of patients with LC are or were smokers (2). Smokers have 20-30 times more risk to developed LC (3). This situation turns LC a disease that can be prevented. National Comprehensive Cancer Network (NCCN) has suggested a smoking cessation program as an integral part of oncological treatment for smokers patients (4). Age is another important risk factor for the development of LC. Average age of patients at LC diagnosis is higher, around 70 years old in US (5) and 63 years old in Brazil $(6,7)$. However, elderly patients with LC are often undertreated for all oncological modalities (8).

Unfortunately, $50 \%$ to $60 \%$ of cases has been diagnosed with metastatic or advanced stage in different countries $(7,9,10)$. Most cured patients were habitually submitted to surgery associated with chemotherapy and/or radiation therapy (RT). But, only $15 \%$ to $20 \%$ of patients diagnosed with non-small cell lung cancer (NSCLC) were treated by surgery. Between $25 \%$ to $30 \%$ of cases of NSCLC are stage IIIA/B, locally advanced and with inoperable disease $(3,5,11)$. Therefore, LC still remains an important challenge for oncology care today with overall survival (OS) 5 years around $15 \%$ of patients.

The rationale behind the chemo and radiation therapy (CRT) association is to have both a better regional and systemic control of disease. The most common cause of mortality in patients with stage III unresectable NSCLC is distant recurrent disease (12). Moreover, CRT could be concurrent (cCRT) or sequential (sCRT), but most trials shown better survival with concurrent association (13). The median progression-free survival among patients who has been treated by CRT is around 8 months and only $20 \%$ of patients are alive at 5 years after NSCLC diagnosis $(11,14,15)$. sCRT could be less toxic but OS has been fallen 6-7\% when compared to cCRT and sCRT has been as alternative option in elderly or low performance patients or with severe co-morbidities (15).

Platinum-based doublet chemotherapy given with cCRT is considered the preferred treatment for selected patients with unresectable early or locally advanced NSCLC (14), because survival is better than compared to sCRT (15). Currently, in spite of advances in technology and treatment, cCRT has been associated with high incidence of significant toxicity (grades 3 or 4), specially, esophagitis and pneumonitis. Therefore, delay or interruptions in either chemotherapy or radiotherapy have been frequently reported (16).

The issue of missed chemotherapy doses during CRT was reported as a factor that worsens the prognosis and increases mortality in the study by Deek et al. in the $A m \mathcal{F}$ Clin Oncol (17). Authors showed that the median OS was 9.6 and 24.3 months, respectively, for patients with missed chemotherapy versus patients without missed chemotherapy. Moreover, when missed chemotherapy was due to poor ECOG performance status (PS), the survival was only 4.6 months. Finally, in multivariate models, the mortality was 1.97 higher in the group that missed chemotherapy. 
This study also reported that the main reasons to miss chemotherapy was hematologic toxicity (59\%), esophagitis $(17 \%)$, decline in PS (12\%) and allergic reaction (5\%). Oddly, age of patients was not reported, what limits the impact and applicability of their data.

RT in chest often causes inflammation of the epithelium of esophagus and this damage increases when chemotherapy is associated with radiation. As a result, cCRT increases esophageal toxicities over sCRT or one modality alone (16). Patients after CRT with symptomatic radiation esophagitis habitually present as dysphagia, odynophagia or reflux-like symptoms, such as epigastric or sternal chest pain. These patients have a high difficulty in feeding, and sometimes nutritional support is required through a nasoenteral probe. Patients with previous history of reflux disease may exacerbate grade of esophagitis (16).

Hematologic toxicities are very common in patients treated by cCRT (16). Because chemotherapy is a systemic modality of treatment that can affect different groups of hematologic cells, rates of grade $>3$ thrombocytopenia, leukopenia and granulocytopenia can reach $10 \%, 70 \%$ and $71 \%$ of patients, respectively. RT on vertebral bone marrow has been understudied for LC patients and could worsen levels of hematologic toxicities (16).

Independent factors of worse prognosis have been identified in patients receiving cCRT for LC stage III. Deek et al. also identified that the decline in PS during cCRT was associated with the worst survival (17). Weight loss and advanced $\mathrm{T}$ stage were associated with worse response, survival and toxicities on the multivariate analyses of 425 patients with LC stage IIIB (18). In an observational population-based study of patients with NSCLC stage III from Belgium and Netherlands the authors identified that higher age and advanced $\mathrm{N}$-stage were much more related with sequency therapy than concurrent therapy (13). Another Korean study identified that age $>75$ years old, diffusion lung capacity for carbon monoxide $\leq 80 \%$, gross tumor volume $\geq 100 \mathrm{~cm}^{3}$ and subcarinal node involvement were associated with poor OS both in univariate and multiunivariate analyses (19).

Time to start treatment after diagnosis of LC has been established as an important cancer care quality measure. Although, timely LC care is important, its real impact on the survival remains unclear (20). Non-simultaneous initiation of CRT also was associated with differences in OS (21). Moreover, patients under cCRT, delay or dose reduction of chemotherapy seems worsens the prognosis (17).

Regarding to the regimens of chemotherapy used in concurrent therapy, the standard of care for patients stage IIIA unresectable have been a platinum-based doublet: the two most frequently regimens used in US were cisplatin-etoposide or carboplatin-paclitaxel. A systematic review analysed these two regimens and they were comparable in terms of efficacy and toxicities showed higher rates of grade $\geq 3$ thrombocytopenia and neutropenia in the regimen carboplatin-paclitaxel. There was no significant difference in response rates, OS, progression-free survival, locoregional relapse, distant metastasis and rates of pneumonitis or esophagitis (11).

Combination CRT with molecular targeting or/and immunotherapy could improve benefits. There are many experimental evidences about a synergistic effect between radiation and immune checkpoints inhibitors, with a very important potential of enhancing immuno-modulating effects and improving resistance (22). Moreover, an effect induced by local RT, called abscopal effect, would create a systemic anti-tumor immune response, with effect over non-irradiated metastatic lesions distant from the site of irradiation (23). Even though this abscopal effect has been reported in many cases and trials, its occurrence rate is low. The mechanism of the abscopal effect is not clear and needs to be better explained and understood. The personalization of cancer therapy, predicated largely on genomic interrogation, is facilitating these lection of therapies that are directed against driver mutations, aberrant cell signaling, tumour microenvironments, and genetic susceptibilities. Molecular targeted agents are also opportunities to improve results of the CRT. Target drugs could replace conventional chemotherapeutic drugs in combined treatments (24).

Local RT and immune therapy association could amplify the anti-tumor immune response in local and systematic controls (23). For improving survival in patients with NSCLC stage III unresectable new drugs and strategies should be tested. Recently, Durvalumab, a human IgG monoclonal antibody that blocks programmed death ligand 1 (PD-L1) binding to programmed cell death protein 1 (PD-1), was tested after cCRT with platinum-based doublet versus placebo. Durvalumab was associated with better progression-free survival (16.8 vs. 5.6 months with placebo). In the group Durvalumab, $15.4 \%$ of patients discontinued the study because drug adverse events versus $9.8 \%$ in the placebo group (25).

On the topic of improving regimens of cCRT, the standard radiation dose was defined for NSCLC as 60 Gy, because of decreased survival in patients treated with 74 Gy (26). Moreover, image-guided RT has become the standard of care in many services, allowing for reduced target 
volume that could decrease toxicities (16). New modalities techniques, as 3D-conformal radiotherapy (3DCRT), 4-dimensional computed tomography (4DCT), intensity modulated radiotherapy (IMRT), and photon therapy have been incorporated recently with the goals to decrease levels of toxicities, lower $V_{20}$ and better OS (27). These modern techniques reduce irradiation in normal areas and improve dose in tumor areas. Besides alternative radiation strategies spanning from dose intensification, use of serial positron emission tomography-computed tomography to select highrisk patients and use proton therapy should be properly tested in well-designed clinical trials.

The RT needs to be better present the era of precision medicine. Genomic studies have shown biological heterogeneity to be a central characteristic of cancer. A gene-expression-based radiosensitivity index as a molecular estimate for cellular survival fraction at 2 Gy (SF2) was identified and joined with linear quadratic model (model that estimates different radiation fractionation schemes with similar clinical effect), was called the genomic-adjusted radiation dose (GARD) (28). A high GARD value predicts for high therapeutic effect for radiotherapy and higher values of GARD was associated with better OS in different kind of solids tumors. Then, individualized radiation dose on the basis of gene-expression profiles reflecting the radiosensitivity of tumour and normal tissue $(24,28)$.

On the subject of elderly patients, so far cCRT have not improved outcome and these patients are more likely to be selected for sCRT in retrospective studies. In fact, median survivals are not significantly different between cCRT or sCRT. However, severe toxicities rate has been higher in older than in young patients. Moreover, most patients in these studies were elderly patients (more than 70 years old) with very good PS (0-1) and limited co-morbidities. Then, more solid knowledge on the best CRT for elderly patients needs further prospective research evaluating different doses (27) and new target drugs or/and immunotherapy (24).

In conclusion, Deek et al. got to show how important is to reduce toxicities to ensure the delivery of all chemotherapy doses in favor of improving survival in patients treated by cCRT. CRT need to be initiated early and concomitant, respecting clinical conditions of patients and adjusted for their best clinical benefits. This is a very important message if we assume that stage III patients represent a delicate cohort where the balance between toxicity, curability and comorbidities must be well balanced. However, the question here is: is clinical care good enough in light of the current knowledge on tumor biology and the novel technologies available? In the era of precision medicine and immune-oncology we can dare to go beyond. Systematic research efforts are being made to facilitate individualized radiation dose on the basis of geneexpression profiles reflecting the radiosensitivity of tumour and normal tissue. This advance in precision radiotherapy should complement those benefits obtained from precision cancer medicine that use molecularly targeted agents and immunotherapies. According to Bristow et al. "With the increasing technical power of radiotherapy to safely increase local tumour control for many solid tumours, it is an opportune time to rigorously explore the potential benefits of combining radiotherapy with molecular targeted agents and immunotherapies to increase cancer survival outcomes". The lessons learned from addition of Durvalumab to CRT may be just the beginning of a new era for cCRT for stage III NSCLC patients. By identifying the potential predictors of the abscopal effect we will be able to select the most appropriate patients who would most likely benefit from the combination treatment modality. So, let's move to the next step.

\section{Acknowledgements}

None.

\section{Footnote}

Conflicts of Interest: The authors have no conflicts of interest to declare.

\section{References}

1. Bray F, Ferlay J, Soerjomataram I. Global Cancer Statistics 2018: GLOBOCAN Estimates of Incidence and Mortality Worldwide for 36 Cancers in 185 Countries. CA Cancer J Clin 2018;68:394-424.

2. Moura MA, Bergmann A, Aguiar SS, et al. The magnitude of the association between smoking and the risk of developing cancer in Brazil: a multicenter study. BMJ Open 2014;4:e003736.

3. Alberg AJ, Brock MV, Ford JG, et al. Epidemiology of lung cancer: Diagnosis and management of lung cancer, 3rd ed: American College of Chest Physicians evidencebased clinical practice guidelines. Chest 2013;143:e1S-29S.

4. Davis J. Smoking Cessation. NCCN.org. 2018.

5. Siegel RL, Miller KD, Jemal A. Cancer statistics, 2018. CA Cancer J Clin 2018;68:7-30.

6. Costa GJ, Mello MJ, Ferreira CG, et al. Increased 
incidence, morbidity and mortality rates for lung cancer in women in Brazil between 2000 and 2014: an analysis of three types of sources of secondary data. Lung Cancer 2018;125:77-85.

7. Costa G, Thuler LC, Ferreira CG. Epidemiological changes in the histological subtypes of 35,018 non-small-cell lung cancer cases in Brazil. Lung Cancer 2016;97:66-72.

8. Costa GJ, de Mello MJ, Ferreira CG, et al. Undertreatment trend in elderly lung cancer patients in Brazil. J Cancer Res Clin Oncol 2017;143:1469-75.

9. Cancer Research UK [homepage on the Internet]. London: Cancer Research UK; [cited 2015 Oct 1]. Lung Cancer Statistics. Available online: http:// cancerresearchuk.org/health-professional/cancer-statistics/ statistics-by-cancer-type/lung-cancer

10. Malvezzi M, Carioli G, Bertuccio P, et al. European cancer mortality predictions for the year 2017, with focus on lung cancer. Ann Oncol 2017;28:1117-23.

11. Steuer CE, Behera M, Ernani V, et al. Comparison of Concurrent Use of Thoracic Radiation With Either Carboplatin-Paclitaxel or Cisplatin-Etoposide for Patients With Stage III Non-Small-Cell Lung Cancer: A Systematic Review. JAMA Oncol 2017;3:1120-9.

12. Salama JK, Vokes EE. New radiotherapy and chemoradiotherapy approaches for non-small-cell lung cancer. J Clin Oncol 2013;31:1029-38.

13. Walraven I, Damhuis RA, Ten Berge MG, et al. Treatment Variation of Sequential versus Concurrent Chemoradiotherapy in Stage III Non-Small Cell Lung Cancer Patients in the Netherlands and Belgium. Clin Oncol (R Coll Radiol) 2017;29:e177-85.

14. Postmus PE, Kerr KM, Oudkerk M, et al. Early and locally advanced non-small-cell lung cancer (NSCLC): ESMO Clinical Practice Guidelines for diagnosis, treatment and follow-up. Ann Oncol 2017;28:iv1-21.

15. Curran WJ Jr, Paulus R, Langer CJ, et al. Sequential vs. concurrent chemoradiation for stage III non-small cell lung cancer: randomized phase III trial RTOG 9410. J Natl Cancer Inst 2011;103:1452-60.

16. Verma V, Simone CB 2nd, Werner-Wasik M. Acute and Late Toxicities of Concurrent Chemoradiotherapy for Locally-Advanced Non-Small Cell Lung Cancer. Cancers (Basel) 2017;9. doi: 10.3390/cancers9090120.

17. Deek MP, Kim S, Ahmed I, et al. Prognostic Impact of Missed Chemotherapy Doses During Chemoradiation Therapy for Non-Small Cell Lung Cancer. Am J Clin Oncol 2018;41:362-6.

18. Topkan E, Parlak C, Selek U. Impact of weight change during the course of concurrent chemoradiation therapy on outcomes in stage IIIB non-small cell lung cancer patients: retrospective analysis of 425 patients. Int J Radiat Oncol Biol Phys 2013;87:697-704.

19. Kim YH, Ahn SJ, Kim YC, et al. Predictive factors for survival and correlation to toxicity in advanced Stage III non-small cell lung cancer patients with concurrent chemoradiation. Jpn J Clin Oncol 2016;46:144-51.

20. Nadpara P, Madhavan SS, Tworek C. Guidelineconcordant timely lung cancer care and prognosis among elderly patients in the United States: A population-based study. Cancer Epidemiol 2015;39:1136-44.

21. Deek MP, Kim S, Beck R, et al. Variations in Initiation Dates of Chemotherapy and Radiation Therapy for Definitive Management of Inoperable Non-Small Cell Lung Cancer Are Associated With Decreases in Overall Survival. Clin Lung Cancer 2018;19:e381-90.

22. Filippi AR, Di Muzio J, Badellino S, et al. Locallyadvanced non-small cell lung cancer: shall immunotherapy be a new chance? J Thorac Dis 2018;10:S1461-7.

23. Liu Y, Dong Y, Kong L, et al. Abscopal effect of radiotherapy combined with immune checkpoint inhibitors. J Hematol Oncol 2018;11:104.

24. Bristow RG, Alexander B, Baumann M, et al. Combining precision radiotherapy with molecular targeting and immunomodulatory agents: a guideline by the American Society for Radiation Oncology. Lancet Oncol 2018;19:e240-51.

25. Antonia SJ, Villegas A, Daniel D, et al. Durvalumab after Chemoradiotherapy in Stage III Non-Small-Cell Lung Cancer. N Engl J Med 2017;377:1919-29.

26. Demedts IK, Vermaelen KY, van Meerbeeck JP. Treatment of extensive-stage small cell lung carcinoma: current status and future prospects. Eur Respir J 2010;35:202-15.

27. Bayman N, Blackhall F, McCloskey P, et al. How can we optimise concurrent chemoradiotherapy for inoperable stage III non-small cell lung cancer? Lung Cancer 2014;83:117-25.

28. Scott JG, Berglund A, Schell MJ, et al. A genomebased model for adjusting radiotherapy dose (GARD): a retrospective, cohort-based study. Lancet Oncol 2017;18:202-11.

Cite this article as: Costa GJ, Ferreira CG, Thuler LC. Concurrent chemoradiotherapy for stage III non-small cell lung cancer: correct clinical management as the basis to move beyond. Ann Transl Med 2018;6(Suppl 1):S65. doi: 10.21037/ atm.2018.10.38 